\title{
Managing thymic enlargement in Graves' disease
}

\author{
C Kamath1', J Witczak², M A Adlan² and L D Premawardhana1,2 \\ ${ }^{1}$ Centre for Endocrine and Diabetes Sciences, University Hospital of Wales, Cardiff, UK and 2Section of Endocrinology, \\ Department of Medicine, Ysbyty Ystrad Fawr, Caerphilly, UK
}

\section{Summary}

Thymic enlargement (TE) in Graves' disease (GD) is often diagnosed incidentally when chest imaging is done for unrelated reasons. This is becoming more common as the frequency of chest imaging increases. There are currently no clear guidelines for managing TE in GD. Subject 1 is a 36-year-old female who presented with weight loss, increased thirst and passage of urine and postural symptoms. Investigations confirmed GD, non-PTH-dependent hypercalcaemia and Addison's disease (AD). CT scans to exclude underlying malignancy showed TE but normal viscera. A diagnosis of hypercalcaemia due to GD and AD was made. Subject 2, a 52-year-old female, was investigated for recurrent chest infections, haemoptysis and weight loss. CT thorax to exclude chest malignancy, showed TE. Planned thoracotomy was postponed when investigations confirmed GD. Subject 3 is a 47-year-old female who presented with breathlessness, chest pain and shakiness. Investigations confirmed T3 toxicosis due to GD. A CT pulmonary angiogram to exclude pulmonary embolism showed TE. The CT appearances in all three subjects were consistent with benign TE. These subjects were given appropriate endocrine treatment only (without biopsy or thymectomy) as CT appearances showed the following appearances of benign TE - arrowhead shape, straight regular margins, absence of calcification and cyst formation and radiodensity equal to surrounding muscle. Furthermore, interval scans confirmed thymic regression of over $60 \%$ in 6 months after endocrine control. In subjects with CT appearances consistent with benign TE, a conservative policy with interval CT scans at 6 months after endocrine control will prevent inappropriate surgical intervention.

\section{Learning points:}

- Chest imaging is common in modern clinical practice and incidental anterior mediastinal abnormalities are therefore diagnosed frequently.

- Thymic enlargement (TE) associated with Graves' disease (GD) is occasionally seen in view of the above.

- There is no validated strategy to manage TE in GD at present.

- However, CT (or MRI) scan features of the thymus may help characterise benign TE, and such subjects do not require thymic biopsy or surgery at presentation.

- In them, an expectant 'wait and see' policy is recommended with GD treatment only, as the thymus will show significant regression 6 months after endocrine control.

\section{Background}

Thymic enlargement (TE) is known to occur both in Graves' disease (GD) and Addison's disease (AD) and in myasthenia gravis. Its incidence is unknown as the thymus is not routinely imaged in them - TE often being diagnosed when imaging is done for unrelated reasons in GD, that is they are 'thymic incidentalomas'. TE in GD was first described more than a century ago (1), and it is plausible that it may be related to its role in endocrine 
Table 1 Treatment of Graves' disease and thymic regression. Reports of subjects who had thionamide therapy for GD (combined with RAl in some) who showed thymic regression on repeat CT scanning.

\begin{tabular}{l}
\hline Author, year of publication \\
\hline Kamath 2017 (current report) \\
Jinguji 2017 (10) \\
Takahashi 2017 (15) \\
Haider 2017 (9) \\
Shanmugasundaram 2016 (16) \\
Song 2016 (17) \\
Betterle 2014 (2) \\
Airel 2013 (18) \\
Popoveniuc 2010 (19) \\
Carvalho 2010 (20) \\
Kubicky 2010 (21) \\
Takami 2009 (22) \\
Tsuda 2008 (23) \\
Giovanella 2008 (24) \\
Inaoka 2007 (25) \\
Van Nieuwkoop 2005 (26) \\
Brinkane 2004 (27) \\
Nakamura 2004 (28) \\
Budavari 2002 (29) \\
Nicolle 1999 (30) \\
Murakami 1996 (31) \\
Ohno 1995 (32)
\end{tabular}

\begin{tabular}{crr}
\hline GD subjects treated & & Thymic regrn \\
\cline { 1 - 2 } 3 & & \\
40 & \\
1 & 10 \\
1 & 1 \\
1 & 1 \\
1 & 1 \\
1 & 1 \\
& \\
1 & 1 \\
3 & 2 \\
1 & 1 \\
1 & 1 \\
1 & 1 \\
1 & 1 \\
1 & 3 \\
18 &
\end{tabular}

1

1

1

2

1

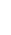

33 subjects showed a decrease in thymic size 6 months after treatment; thymic size was stable in the rest (mean follow up - 21.9 months) both regressed with thionamides

Thymic regression during thionamide therapy and expansion during thionamide free periods

The thymus regressed after thionamide therapy but enlarged again when GD relapsed

Associated with pericarditis

Only 2 subjects returned for follow up
6 had RAl as primary therapy; 34 had thionamides and RAI Submandibular ectopic thymus, and normal thymus -

Only 13 subjects had CT scans before and after thionamide therapy autoimmunity. Several further reports of TE in GD have been published since then (Table 1).

There are no controlled studies about the nature of TE and its outcome in GD. This is important as although the vast majority of TE is benign, as evidenced by regression during treatment or by histology following surgery, malignancy may occur in a small minority (2). Several studies indicate partial or complete regression in the majority after thionamide treatment (Table 1). This paucity of controlled evidence causes therapeutic uncertainty when TE complicates GD. Also, there are no controlled outcome studies comparing thymic biopsy/ surgery at presentation to an expectant 'wait and see' policy of GD treatment and interval scanning. Physicians looking for a safe management pathway often find radiological features of the thymus (e.g. CT or MRI scan appearances) useful in this regard $(3,4)$.

We describe three patients with TE in GD. They were identified as having likely benign TE on CT appearances, given specific endocrine therapy only and had interval scans 6 months after control. Significant thymic regression occurred in all. Thus, biopsy and/or surgery was avoided in all three subjects.

\section{Case presentation}

\section{Subject 1}

A 36-year-old woman presented with recent onset vomiting, thirst, constipation, muscle aches and dehydration. She had an unintentional weight loss of five stones in 4 months, with hair loss, 'shakiness' and postural symptoms. She took no medication and did not smoke or consume alcohol. Clinical examination showed a dry tongue, a regular pulse of 100/min, tremors, alopecia, and a smooth, symmetrical goitre with a loud bruit. She also had periorbital oedema but no signs of active Graves' orbitopathy (GO). She had no buccal or skin pigmentation and systems examination was normal. After adequate intravenous fluid therapy, she had persistent postural hypotension - blood pressure of 115/51 lying and $91 / 50 \mathrm{mmHg}$ on standing.

\section{Subject 2}

A 52-year-old nurse was referred for hyperthyroidism first noticed during pre-operative assessment for thymic biopsy. She presented with recurrent chest 
infections, haemoptysis and an unintentional weight loss of $15 \mathrm{~kg}$ over a few months. She had the sickle cell trait, hypertension and tuberculous uveitis and was on lanosprazole, tramadol, amlodipine and propylthiouracil. Clinical examination showed a firm, symmetrical goitre, with no bruit or GO. Systems examination was normal.

\section{Subject 3}

A 47-year-old, previously well woman, presented acutely with chest pain and breathlessness of several weeks' duration with lack of energy and 'shakiness'. She had no weight loss or eye symptoms. She had deep vein thrombosis and depression in the past but took no medication. She was a non-smoker who consumed 2-4 units of alcohol each week. She had a regular pulse of
$88 / \mathrm{min}$, blood pressure of $151 / 88 \mathrm{mmHg}$ and normal systems examination. She had no goitre or GO at the time of review in the Endocrine clinic.

\section{Investigation}

\section{Subject 1}

Investigations - (a) free T3: $>46.1 \mathrm{pmol} / \mathrm{L}$ (2.6-5.7), free T4: $59.5 \mathrm{pmol} / \mathrm{L}$ (9.0-19.1), TSH: <0.01 mU/L (0.30-4.40); (b) thyrotrophin receptor antibody (TRAb): $25.5 \mathrm{U} / \mathrm{L}$ (<1.6 U/L); (c) corrected calcium: $2.98 \mathrm{mmol} / \mathrm{L}$ (2.20-2.60); (d) PTH: $<0.5 \mathrm{pmol} / \mathrm{L}$ (1.6-7.2); (e) short Synacthen test, cortisol 305 (0 min) and $343 \mathrm{nmol} / \mathrm{L}$ (30 min); (f) adrenal antibodies positive; (g) angiotensin-converting enzyme: 94U/L (<52); (h) CT scans (to exclude malignancy) showed TE (Fig. 1A) but normal viscera.
A

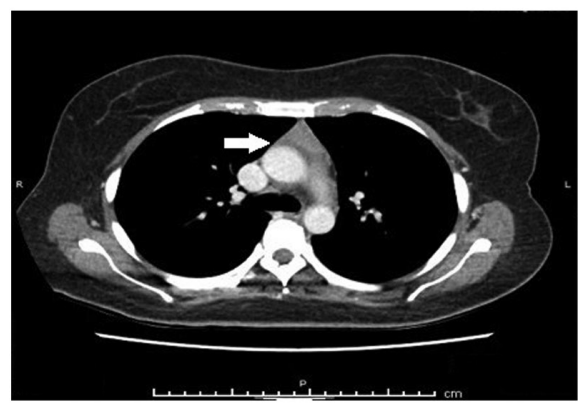

B
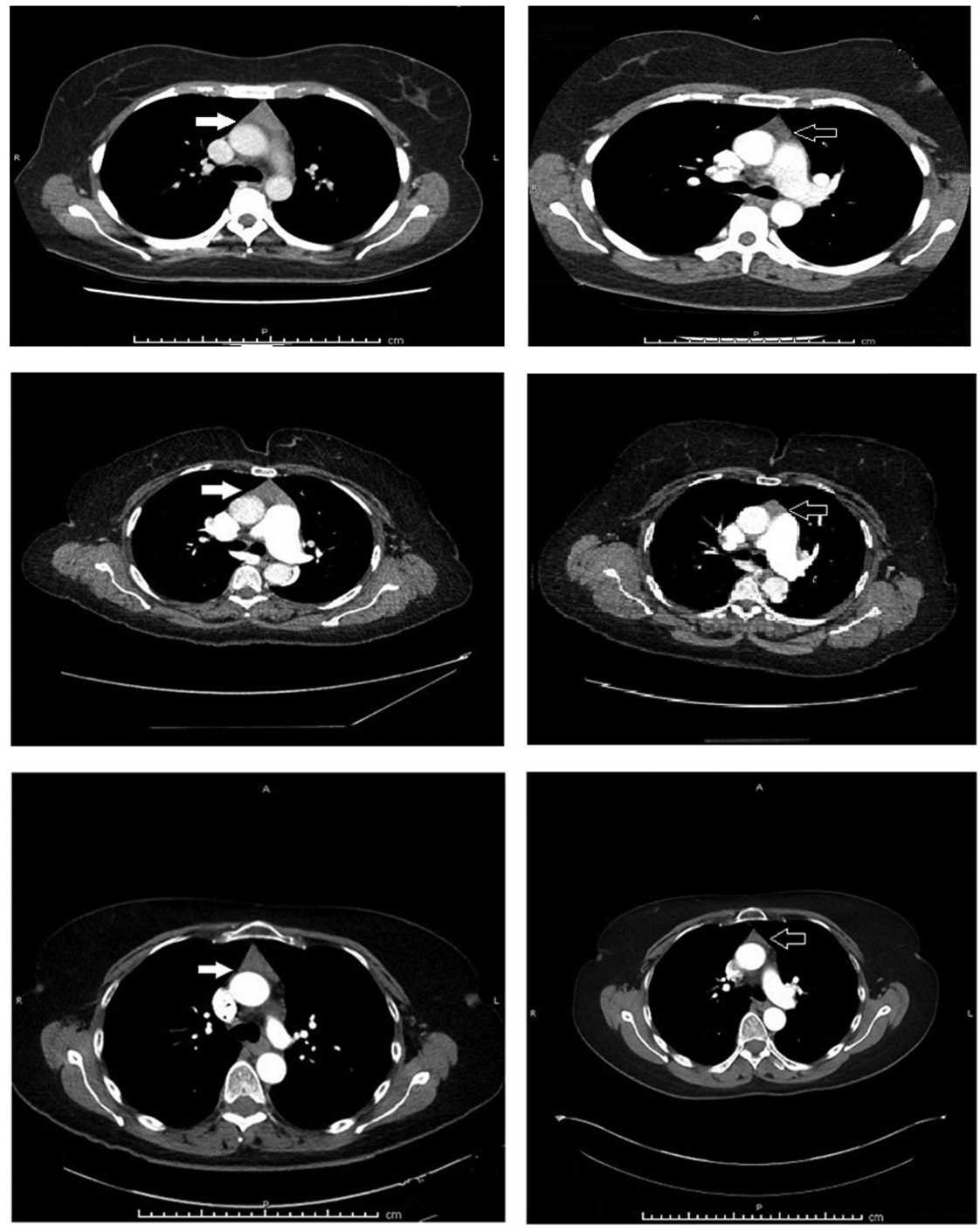

Figure 1

CT scan images of the thymus in three subjects, before and 6 months after thionamide treatment. CT scans of three subjects with Graves' disease (GD) who had thymic enlargement diagnosed incidentally, showing benign characteristics of the thymus - triangular shape, regular straight borders, homogeneous opacification and no invasion of surrounding structures, calcification or cyst formation (A - left hand panels - solid white arrows). Six months after thionamide therapy for $\mathrm{GD}$, there has been significant regression of the thymus (in excess of $60 \%$ in volume), confirming their benign nature ( $B$ - right hand panels - solid black arrows with white border). 
A diagnosis of non-PTH-mediated hypercalcaemia, complicating GD and AD was made.

\section{Subject 2}

Investigations - (a) free T4: $44.4 \mathrm{pmol} / \mathrm{L}$, free $\mathrm{T} 3$ : $36.9 \mathrm{pmol} / \mathrm{L}$, TSH: $<0.02 \mathrm{mU} / \mathrm{L}$; (b) TRAb: $3.6 \mathrm{U} / \mathrm{L}$; (c) alkaline phosphatase: 208 (30-150U/L); gamma GT: $27 \mathrm{U} / \mathrm{L}$ (9-36); (d) vitamin D: $14 \mathrm{nmol} / \mathrm{L}$ ( $<30$ is deficient); (e) PTH: $14.1 \mathrm{pmol} / \mathrm{L}$; (f) serum calcium: $2.28 \mathrm{mmol} / \mathrm{L}$; (g) CT thorax (done to exclude malignancy) showed TE (Fig. 1A). GD was diagnosed and treatment was changed to carbimazole.

\section{Subject 3}

Investigations - (a) free T3: $6.7 \mathrm{pmol} / \mathrm{L}$; free $\mathrm{T} 4$ : $18.7 \mathrm{pmol} / \mathrm{L}$; TSH: $<0.02 \mathrm{mU} / \mathrm{L}$; (b) TRAb: $4.5 \mathrm{U} / \mathrm{L}$; (c) chest X'ray - normal; (d) CT pulmonary angiogram to exclude pulmonary embolism (PE), showed no PE but demonstrated TE (Fig. 1A). A diagnosis of T3 toxicosis due to GD was made.

\section{Treatment}

\section{Subject 1}

She was rapidly rehydrated and given $4 \mathrm{mg}$ of zoledronic acid intravenously. Carbimazole and selenium were given for GD and mild inactive GO, and hydrocortisone (HC) and fludrocortisone (FC) for $\mathrm{AD}$, with significant symptomatic improvement.

\section{Subject 2}

Thoracic surgery was cancelled. She is currently on carbimazole and vitamin D and clinically and biochemically euthyroid.

\section{Subject 3}

She was commenced on carbimazole in appropriate doses.

\section{Outcome and follow-up}

\section{Subject 1}

CT thorax 6 months later showed a $67 \%$ reduction in thymic volume - from $38 \times 15 \times 51 \mathrm{~mm}$ to $23 \times 11 \times 38 \mathrm{~mm}$ (Fig. 1B). More than 1 year later, she is asymptomatic without postural hypotension and her calcium levels have remained normal. She is also clinically and biochemically euthyroid.

\section{Subject 2}

CT thorax, 6 months after endocrine control, showed a $65 \%$ reduction in thymic volume - from $63 \times 59 \times 22 \mathrm{~mm}$ to $42 \times 34 \times 20 \mathrm{~mm}$ (Fig. 1B). Her vitamin D and liver enzymes have normalised and 1 year later she is clinically and biochemically euthyroid.

\section{Subject 3}

CT chest 6 months after endocrine control showed a $61 \%$ reduction in thymic volume (Fig. 1B) - from $41 \times 18 \times 57 \mathrm{~mm}$ to $31 \times 13 \times 41 \mathrm{~mm}$. Her GD remains well controlled.

All three subjects had thymic 'incidentalomas' at presentation. We managed them with specific treatment for GD only (and AD in subject 1), as the CT features were consistent with benign TE (Fig. 1A). Repeat CT scans 6 months after endocrine control confirmed thymic regression (Fig. 1B). We prevented planned thoracotomy in subject 2 .

\section{Discussion}

We have described three subjects with TE with GD. They all had thymic 'incidentalomas' on CT scans done for unrelated reasons. We adopted a 'wait and see' approach because the radiological features of the thymus were consistent with benign enlargement - thus avoiding transthoracic biopsy and/or surgery. All three subjects showed significant thymic regression (between 61 and $67 \%$ by volume) 6 months after endocrine control.

\section{The utility of CT scans in TE with GD}

The thymus is largest and radiologically most prominent at puberty and then becomes smaller and replaced by fat (4). Its radiodensity declines in comparison to surrounding muscle (4). As chest radiology is increasingly done for unrelated reasons as in our subjects (screening for malignancy, investigating chest pain and breathlessness), mediastinal abnormalities are more commonly seen. Approximately $0.4 \%$ of smokers over the age of 40 years, and $0.9 \%$ of the Framingham Heart Study population, had anterior mediastinal masses $(5,6)$.

The utility of CT imaging in differentiating between benign TE and malignancy is becoming clearer, although 
no imaging features absolutely differentiate between the two. In one study examining 124 CT scans of subjects who underwent thymic surgery, shape, straight clear margins, homogeneous attenuation, absent lymphadenopathy or invasion of surrounding structures, were identified as features important in differentiation (3). Most authorities agree that benign TE is characterised by (a) an arrowhead, triangular or bipyramidal appearance with straight regular margins, (b) radiodensity equal to surrounding thoracic muscles, (c) lack of infiltration of surrounding structures and (d) lack of calcification and cyst formation (3). Significant thymic regression after endocrine control is also good evidence of benign TE. MRI scans may be useful to differentiate between the two when in doubt (techniques utilise the lower fat content of thymic tumours compared to the higher fat content of benign hyperplasia).

Our three subjects fulfilled both sets of criteria (a) 'benign' thymic CT appearances and (b) significant reduction in thymic volume (61-67\%) 6 months after GD control (Fig. 1B).

\section{Regression of thymic enlargement during treatment of GD}

There is good evidence that significant regression of TE occurs with GD treatment confirming its benign nature in most $(2,7,8)$. Betterle et al. reported 107 cases of TE complicating GD, of whom only four were malignant (2). Murakami et al. in an early study, compared thymic CT in 13 patients with GD, and confirmed a significant reduction of size and radiodensity. Yacoub et al. compared eight subjects who underwent surgery for anterior mediastinal masses in GD, with 13 subjects who were given drugs only with interval scanning. All surgical samples were histologically benign and thymic regression occurred in all who had only GD therapy (8). There is also evidence of thymic regression in a review of 31 case reports of GD subjects who only had drugs for GD followed by interval CT scans (9). More recently, Shanmugasundaram et al. published a report of a single patient and Jinguji et al. published a case series of 40 subjects who had thionamides and RAI, where thymic regression followed therapy for GD (10). These data suggest that the vast majority of TE in GD is benign (103/107 in Betterle's series) and that thionamide therapy either alone or followed by RAI results in thymic regression. Many surgeons now question the need for surgery on thymic 'incidentalomas' associated with GD, despite improvement in surgical technique and minimally invasive thymectomies.

\section{Incidence and possible mechanisms of TE}

The incidence of TE in GD and $\mathrm{AD}$ is unknown and a study examining this is unlikely any time soon. However, there have been many case reports and series describing this relationship (Table 1). The mechanisms for TE in GD are not clear either. (a) Thyroid hormones - Early animal studies showed thymic regression after thyroidectomy and an increase in thymic size and weight on thyroxine treatment. Thyroxine induced changes to the thymic cortex were demonstrated in humans later. (b) Thyroid autoimmunity and angiogenesis - The thymus has functional TSH receptors (TSHR) - Kim et al. found TSHR in $82 \%$ of 22 normal thymus glands. It is plausible that TRAb induces thymic growth in a manner analogous to thyroid enlargement in GD. A role for angiogenesis has been proposed as angiogenic factors such as VEGF, Ang-1 and 2 and Tie- 2 found in higher concentration in GD may produce increased goitre volumes and vascularity. These regress upon treatment of GD.

Although TE has been well documented in GD, its incidence in Hashimoto's thyroiditis (HT) is unknown. Early descriptions of histologic thymic hyperplasia in HT (11), and myasthenia gravis and HT occurring sometimes with other autoimmune diseases (12), has been supplemented with occasional case reports in the more recent literature $(13,14)$. No definitive relationship can be deduced from the above.

However, a word of caution - (a) there are no absolute radiological criteria that differentiate between benign and potentially malignant TE; (b) although CT scanning is used as standard imaging for characterisation, there has been no comparison with MRI or PET scanning; (c) there is no consensus with regard to the length of follow-up before repeating CT after endocrine control - most advocating 3-6 months; (d) there is no evidence about the superiority of different surgical approaches (biopsy vs thymectomy), compared to a 'wait and see' policy as no comparison has been done.

\section{Conclusions and recommendations}

We have described incidentally diagnosed TE in three subjects with GD who had scans for unrelated reasons. We repeated scans 6 months after endocrine control and showed thymic volume regression of $61-67 \%$. The incidence of TE in endocrine disorders is unclear and controlled studies are lacking about the management of this condition. CT imaging may help differentiate between benign TE and likely malignant TE, although 
there are no absolute certainties. MRI scans may be used to clarify. Those selected for a non-surgical approach with thionamide therapy alone should undergo a repeat CT (or MRI) scan in 6 months after endocrine control. All benign lesions would undergo regression with significant change in thymic volume. However, if there is any doubt clinically or radiologically, a biopsy or thymectomy should be considered until conclusive evidence to a different approach becomes available.

\section{Declaration of interest}

The authors declare that there is no conflict of interest that could be perceived as prejudicing the impartiality of the research reported.

\section{Funding}

This study did not receive any specific grant from any funding agency in the public, commercial or not-for-profit sector.

\section{Patient consent}

Written informed consent on the EDM Case Report Journal's consent form has been obtained from the three patients for publication of the submitted article and accompanying images.

\section{Author contribution statement}

C Kamath - Endocrine Speciality Registrar - involved in investigating and management of patients, helped write and review manuscript. J Witzcak Endocrine Speciality Registrar - involved in investigating and management of patients, helped write and review manuscript. M A Adlan - Consultant Physician - member of Endocrine MDT and involved in managing these patients, writing and review of manuscript. L D Premawardhana Consultant Physician - named physician for all three patients, writing of manuscript.

\section{References}

1 MacCallum WG. The pathology of exophthalmic goitre. JAMA 1907 XLIX 1158-1162. (https://doi.org/10.1001/ jama.1907.25320140004001a)

2 Dalla Costa M, Mangano FA \& Betterle C. Thymic hyperplasia in patients with Graves' disease. Journal of Endocrinological Investigation 201437 1175-1179. (https://doi.org/10.1007/s40618-014-0157-7)

3 Ackman JB, Versoza S, Kovach AE, Louissaint A Jr, Lanuti M, Wright CD, Shepard JO \& Halpern EF. High rate of unnecessary thymectomy and its cause; can computed tomography distinguish thymoma, lymphoma, thymic hyperplasia and thymic cysts? European Journal of Radiology 201584 524-533. (https://doi. org/10.1016/j.ejrad.2014.11.042)

4 Araki T, Nishino M, Gao W, Dupuis J, Hunninghake GM, Murakami T, Washko GR, O'Connor GT \& Hatabu H. Normal thymus in adults: appearance on CT and associations with age, sex, BMI and smoking. European Radiology 201626 15-24 (https://doi. org/10.1007/s00330-015-3796-y)

5 Henschke CI, Lee IJ, Wu N, Farooqi A, Khan A, Yankelevitz D \& Altorki NK. CT screening for lung cancer, prevalence and incidence of mediastinal masses. Radiology 2006239 586-590. (https://doi. org/10.1148/radiol.2392050261)

6 Araki T, Nishino M, Gao W, Dupuis J, Washko GR, Hunninghake GM, Murakami T, O'Connor GT \& Hatabu H. Anterior mediastinal masses in the Framingham Heart Study; prevalence and CT image characteristics. European Journal of Radiology 20152 26-31. (https://doi.org/10.1016/j.ejro.2014.12.003)

7 Fabris N, Mocchegiani E, Marrioti S, Paccini F \& Pinchera A. Thyroid function modulates thymic endocrine activity. Journal of Clinical Endocrinology and Metabolism 198662 474-478. (https://doi. org/10.1210/jcem-62-3-474)

8 Figueroa-Vega N. Serum levels of angiogenic molecules in autoimmune thyroid diseases and their correlation with laboratory and clinical features. Journal of Clinical Endocrinology and Metabolism 200994 1145-1153. (https://doi.org/10.1210/jc.2008-1571)

9 Haider U, Richards P \& Gainoukakis AG. Thymic hyperplasia associated with Graves' disease: pathophysiology and proposed management algorithm. Thyroid 201727 994-1000. (https://doi. org/10.1089/thy.2017.0086)

10 Jinguji M, Nakajo M, Nakajo M, Koriyama C \& Yoshiura T. Thymic involution after radioiodine therapy for Graves' disease: relationships with serum thyroid hormones and TRAb. Journal of the Endocrine Society 20171 852-860. (https://doi.org/10.1210/js.2017-00182)

11 Shimabukur S, Tsuchiya M, Mizuno Y, Kurita \& Yoshimatsu H. Thymus in Hashimoto's disease and focal chronic lymphoid thyroiditis. Keio Journal of Medicine 197524 275-287. (https://doi. org $/ 10.2302 / \mathrm{kjm} .24 .275)$

12 Rose $\mathrm{N}$. The role of the thymus in autoimmune thyroid disease. Annals of the New York Academy of Sciences 1975249 116-124. (https://doi.org/10.1111/j.1749-6632.1975.tb29062.x)

13 Khan S, Nunez CP, Narula HS, McLarty A, Bilfinger T \& Carlson HE. Thymic hyperplasia in autoimmune thyroid disease. Endocrine Society 2014, Abstract. Presentation Sat 0500.

14 Bernard C, Frih H, Pasquet F, Kerever S, Jamilloux Y, Tronc F, Guibert B, Isaac S, Devouassoux M, Chalabreysse L, et al. Thymoma associated with autoimmune disease: 85 cases and literature review. Autoimmunity Reviews 201615 82-92. (https://doi.org/10.1016/j. autrev.2015.09.005)

15 Takahashi H, Minami M, Masumoto T, Hiyama T, Kujiraoka Y, Nakamagoe M \& Minami Y. Adult-onset hyperplasia of an ectopic cervical thymus associated with uncontrolled hyperthyroidism: a case report. Iranian Journal of Radiology 201714 e39523.

16 Shamnugasundaram N, Valamparampil JJ, Scott JX, ViJ M \& Narasimhan G. Case reports. BMJ 201611 159-166.

17 Song YS, Won J, Kim MJ, Lee JH, Kim DW, Chung JK, Park do J \& Park YJ. Graves' patient with thymic expression of thyrotropin receptors and dynamic changes in thymic hyperplasia proportional to Graves' disease activity. Yonsei Medical Journal 201657 795-798. (https://doi.org/10.3349/ymj.2016.57.3.795)

18 Airel PS, Steele MB, Lin AH, Seidensticker DF \& Shwayhat AF. Pericarditis, thymic hyperplasia, and Graves' thyrotoxicosis: case report and review of the literature. Military Medicine 2013178 e865-e869. (https://doi.org/10.7205/MILMED-D-12-00531)

19 Popoveniuc G, Sharma M, Devdhar M, Wexler JA, Carroll NM, Wartofsky L \& Burman KD. Graves' and thymic hyperplasia: the relationship of thymic volume to thyroid function. Thyroid $2010 \mathbf{2 0}$ 1015-1018. (https://doi.org/10.1089/thy.2009.0383)

20 Carvalho MR, Dias T, Baptista F \& Do Carmos I. Graves' disease and massive thymic hyperplasia. Thyroid 201020 227-229. (https://doi. org/10.1089/thy.2009.0251)

21 Kubicky RA, Faerber EN, De Chadarevian JP, Wu S, Rezvani I \& De luka F. An adolescent with a mediastinal mass, diagnosed with Graves disease and thymic hyperplasia. Pediatrics 2010125 e433-e437. (https://doi.org/10.1542/peds.2009-2204)

22 Takami M, Omiya H, Higashiyama M, Maeda J, Okami J, Oda K, Tsujinaka T \& Kodama K. A case report of a large thymic 
hyperplasia associated with hyperthyroidism. Annals of Thoracic and Cardiovascular Surgery 200915 404-407.

23 Tsuda E, Imai T, Matsumara A, Hisahara S, Nonaka M, Shiraishi H, Motomura M \& Shimohama S. Thyrotoxic myopathy mimicking myasthenic syndrome associated with thymic hyperplasia. Internal Medicine 200847 445-457. (https://doi.org/10.2169/ internalmedicine.47.0682)

24 Giovanella L, Suriano S \& Ceriani L. Graves' disease, thymus enlargement and hypercalcaemia. New England Journal of Medicine 2008358 1078-1079. (https://doi.org/10.1056/ NEJMc0707309)

25 Inaoka T, Takahashi K, Mineta M, Yamada T, Shuke N, Okizaki A, Nagasawa K, Sugimori H \& Aburano T. Thymic hyperplasia and thymus gland tumours: differentiation with chemical shift MR imaging. Radiology 2007243 869-876. (https://doi.org/10.1148/ radiol.2433060797)

26 Van Nieuwkoop C \& Bolk JH. A mediastinal mass. Graves' disease related thymic hyperplasia. European Journal of Internal Medicine 2005 16 606-607. (https://doi.org/10.1016/j.ejim.2005.03.010)

27 Brinkane A, Ounadi-Corbille W, Bellamy J \& Leroy-Terquen E. Hyperplasia of the thymus in Graves' disease. A case report. Revue de Pneumologie Clinique 200460 239-241. (https://doi.org/10.1016/ S0761-8417(04)72107-0)

28 Nakamura T, Murakami M, Horiguchi H, Nagasaka S, Ishibashi S, Mori M \& Ishikawa SE. A case of thymic enlargement in hyperthyroidism in a young woman. Thyroid 200414 307-310. (https://doi.org/10.1089/105072504323030979)

29 Budavari AI, Whitaker MD \& Helmers RA. Thymic hyperplasia presenting as anterior mediastinal mass in 2 patients with Graves' disease. Mayo Clinic Proceedings 200277 495-499. (https://doi. org/10.1016/S0025-6196(11)62220-9)

30 Nicolle MW. Pseudo-myasthenia gravis and thymic hyperplasia in Graves' disesase. Canadian Journal of Neurological Sciences 199926 201-203. (https://doi.org/10.1017/S0317167100000251)

31 Murakami M, Hosoi Y, Negishi T, Kamiya Y, Miyashita K, Yamada M, Iriuchijima T, Yokoo H, Yoshida I, Tsushima Y, et al. Thymic hyperplasia in patients with Graves' disease. Identification of thyrotrophin receptors in human thymus. Journal of Clinical Investigation 199698 2228-2234. (https://doi.org/10.1172/ JCI119032)

32 Ohno K. A case of thymic enlargement with hyperthyroidism. Nihon Kyobu Shikkan Gakkai Zasshi 199533 785-788.

Received in final form 27 November 2018

Accepted 9 January 2019 